\title{
A dual Yamada-Watanabe theorem for Lévy driven stochastic differential equations*
}

\author{
David Criens ${ }^{\dagger}$
}

\begin{abstract}
We prove a dual Yamada-Watanabe theorem for one-dimensional stochastic differential equations driven by quasi-left continuous semimartingales with independent increments. In particular, our result covers stochastic differential equations driven by (time-inhomogeneous) Lévy processes. More precisely, we prove that weak uniqueness, i.e. uniqueness in law, implies weak joint uniqueness, i.e. joint uniqueness in law for the solution process and its driver.
\end{abstract}

Keywords: Yamada-Watanabe theorem; Lévy process; stochastic differential equation; joint uniqueness; weak uniqueness; weak existence; strong uniqueness; strong existence; martingale problem.

MSC2020 subject classifications: $60 \mathrm{H} 10 ; 60 \mathrm{G} 51 ; 60 \mathrm{H} 05$.

Submitted to ECP on October 22, 2020, final version accepted on February 19, 2021.

\section{Introduction}

The classical Yamada-Watanabe theorem [22] for Brownian stochastic differential equations (SDEs) tells us that strong uniqueness (i.e. pathwise uniqueness) and weak existence implies weak uniqueness (i.e. uniqueness in law) and strong existence. Jacod [11] lifted this result to SDEs driven by semimartingales and extended it by showing that strong uniqueness and weak existence is equivalent to weak joint uniqueness and strong existence. For Brownian SDEs, results related to Jacod's theorem have also been shown by Engelbert [6].

For a moment consider the SDE

$$
d X_{t}=\sigma_{t}(X) d L_{t}, \quad L=\text { Lévy process, }
$$

with non-degenerate coefficient $\sigma$, i.e. $\sigma \neq 0$. Since in this case

$$
d L_{t}=\frac{\sigma_{t}(X) d L_{t}}{\sigma_{t}(X)}=\frac{d X_{t}}{\sigma_{t}(X)},
$$

the noise $L$ can be recovered from the solution $X$ and consequently, weak uniqueness implies weak joint uniqueness. By Jacod's theorem, this observation shows that weak uniqueness and strong existence implies strong uniqueness and weak existence, which

\footnotetext{
${ }^{*}$ Financial support from the DFG project No. SCHM 2160/15-1 is gratefully acknowledged.

${ }^{\dagger}$ Albert-Ludwigs University of Freiburg, Germany.

E-mail: david.criens@stochastik.uni-freiburg.de
} 
can be seen as a dual Yamada-Watanabe theorem. It is a natural and interesting question whether the dual theorem also holds for SDEs with possibly degenerate coefficients, i.e. in case $\sigma=0$ is allowed. Cherny [5] answered this question affirmatively for Brownian SDEs by proving that weak uniqueness implies weak joint uniqueness.

More recently, the dual theorem has been generalized to various infinite-dimensional Brownian frameworks, see [19, 20, 21]. A version for SDEs with Lévy drivers seems to be missing in the literature. To the best of our knowledge, the only formulation of a dual theorem for SDEs with discontinuous noise is the main result of [23], which is a version for SDEs driven by time-inhomogeneous Poisson processes. However, the proof in [23] has a gap. ${ }^{1}$

The purpose of this short paper is to close the gap in the literature and to prove a dual theorem for SDEs driven by quasi-left continuous semimartingales with independent increments (SIIs), which is a large class of drivers including in particular all Lévy processes. We now directly formulate our main result, where we refer to the next section for precise definitions. Let $\mu$ and $\sigma$ be real-valued predictable processes on the path space of càdlàg functions $\mathbb{R}_{+} \rightarrow \mathbb{R}$ and let $L$ be an SII, which we parameterize below by its deterministic semimartingale characteristics.

Theorem 1.1. For the SDE

$$
d X_{t}=\mu_{t}(X) d t+\sigma_{t}(X) d L_{t}, \quad X_{0}=x_{0} \in \mathbb{R},
$$

the following are equivalent:

(i) Strong uniqueness and weak existence holds.

(ii) Weak uniqueness and strong existence holds.

(iii) Weak joint uniqueness and strong existence holds.

We shortly comment on the proof of Theorem 1.1. The implication (i) $\Rightarrow$ (ii) is the classical Yamada-Watanabe theorem and the equivalence of (i) and (iii) is Jacod's theorem. Thus, it suffices to prove (ii) $\Rightarrow$ (iii). More precisely, we prove that weak uniqueness implies weak joint uniqueness, see Theorem 2.3 below. Similar to Cherny's proof for Brownian SDEs, the main idea is to recover the driver $L$ from the solution process $X$ and an independent SII $V$. The technical core of the argument is the proof for independence of $X$ and $V$. We first show that the laws of $X$ and $V$ can be characterized via certain martingale problems and that the covariation between the corresponding test martingales vanishes. This observation allows us to deduce independence from the weak uniqueness assumption with a change of measure argument, which is inspired by ideas from [9]. Our proof for the independence of $X$ and $V$ is different from Cherny's argument, which uses a second auxiliary process. The proofs of the dual theorems in the recent works $[19,20,23]$ are based on Cherny's argument, while in different settings, and the proof in [21] uses results from [20] and the so-called method of the moving frame.

Let us also mention an interesting follow up question: It is well-known that the Yamada-Watanabe theorem remains true for SDEs driven by a Poisson random measure, see [10, Theorem 14.94]. Thus, it is only natural to ask whether the dual theorem also holds for such SDEs. We leave this question for future research.

\footnotetext{
${ }^{1}$ The first sentence in the proof of [23, Lemma 3.3] is the following: "By Lemma 2.3, we only need to prove that two Poisson processes are independent if and only if their sum is also a Poisson process." The if implication, which is supposed to prove a direction of [23, Lemma 3.3] used in the proof of the main result [23, Theorem 3.1], is not true. Indeed, Jacod [13] gave an example of two dependent Poisson processes whose sum is also a Poisson process. The gap in the proof of [23, Lemma 3.3] is that the factorization $E\left[e^{i u(X+Y)}\right]=E\left[e^{i u X}\right] E\left[e^{i u Y}\right]$ does not suffice to conclude independence of $X$ and $Y$.
} 
Finally, we end the introduction with a short comment on our notation: As far as possible, we use the notation developed in the monograph [12] by Jacod and Shiryaev. In particular, we use the Strasbourg notation for (stochastic) integrals, i.e. we write $H \cdot X$ for the stochastic integral of $H$ with respect to $X$. Furthermore, we stress that throughout the paper equalities hold up to a null set.

The article is structured as follows: In Section 2 we provide definitions and the statement that weak uniqueness implies weak joint uniqueness. The proof of this claim is given in Section 3.

\section{The Setting and Main Results}

In this section we introduce our setting in a precise manner. Let us start with a description of the random driver for the SDE (1.2). A real-valued càdlàg adapted process with initial value zero is called SII, if it is a quasi-left continuous semimartingale whose semimartingale characteristics $(B, C, \nu)$ (relative to a fixed truncation function $h: \mathbb{R} \rightarrow \mathbb{R}$ ) are deterministic. It is well-known ([12, Proposition II.2.9]) that the semimartingale characteristics $(B, C, \nu)$ of an SII admit a decomposition

$$
d B_{t}=b_{t} d A_{t}, \quad d C_{t}=c_{t} d A_{t}, \quad \nu(d x, d t)=F_{t}(d x) d A_{t},
$$

for an increasing continuous function $A: \mathbb{R}_{+} \rightarrow \mathbb{R}_{+}$starting in the origin, $d A_{t}$-integrable Borel functions $b: \mathbb{R}_{+} \rightarrow \mathbb{R}$ and $c: \mathbb{R}_{+} \rightarrow \mathbb{R}_{+}$, and a Borel transition kernel $F$ from $\mathbb{R}_{+}$ into $\mathbb{R}$ with $F_{t}(\{0\})=0$ and $\int_{0}^{*} \int\left(1 \wedge|y|^{2}\right) F_{s}(d y) d A_{s}<\infty$. We call such a quadruple $(b, c, F ; A)$ local characteristics of the SII. Clearly, the local characteristics are not unique. Nevertheless, it is well-known ([12, Theorem II.4.25]) that the law of an SII is fully characterized by its semimartingale characteristics and therefore also by its local characteristics. The class of SIIs can equivalently be defined as the class of quasi-left continuous semimartingales with independent increments, see [12, Theorem II.4.15]. This characterization explains the abbreviation SII which stands for semimartingale with independent increments. It is clear that a Lévy process with (time-independent) Lévy-Khinchine triplet $(b, c, F)$ is an SII and (a version of) its local characteristics is given by $\left(b, c, F ; A_{t}=t\right)$.

Next, we introduce the coefficients of the SDE (1.2). Let $\mathbb{D}$ be the Skorokhod space, i.e. the space of càdlàg functions $\mathbb{R}_{+} \rightarrow \mathbb{R}$. We denote the coordinate process by $X$, i.e. $\mathbf{X}(\omega)=\omega$ for all $\omega \in \mathbb{D}$. Further, on $\mathbb{D}$ we define $\sigma$-field $\mathcal{D} \triangleq \sigma\left(\mathbf{X}_{t}, t \in \mathbb{R}_{+}\right)$and the filtration $\mathbf{D} \triangleq\left(\mathcal{D}_{t}\right)_{t \geq 0}$, where $\mathcal{D}_{t} \triangleq \bigcap_{s>t} \sigma\left(\mathrm{X}_{r}, r \in[0, s]\right)$. For a probability measure $P$ on $(\mathbb{D}, \mathcal{D}), \mathbf{D}^{P}$ denotes the $P$-completion of the filtration $\mathbf{D}$.

Throughout the paper, we fix two real-valued $\mathbf{D}$-predictable processes $\mu$ and $\sigma$ on $\mathbb{D}$, an initial value $x_{0} \in \mathbb{R}$ and local characteristics $(b, c, F ; A)$.

We are in the position to define existence and uniqueness concepts for the SDE (1.2). Hereby, we adapt terminology from [5, 11].

Definition 2.1. (i) We call $(\mathbb{B}, L)$ a driving system, if $\mathbb{B}=\left(\Omega, \mathcal{F},\left(\mathcal{F}_{t}\right)_{t \geq 0}, P\right)$ is a filtered probability space with right-continuous and complete filtration which supports an SII $L$ with local characteristics $(b, c, F ; A)$.

(ii) Let $(\mathbb{B}, L)$ be a driving system. We call a càdlàg adapted process $X$ on $\mathbb{B}$ a solution process to the $S D E(1.2)$, if

$$
X=x_{0}+\int_{0}^{\cdot} \mu_{s}(X) d s+\sigma(X) \cdot L,
$$

where it is implicit that the integrals are well-defined, i.e. a.s. $Z_{t} \circ X<\infty$ for 
all $t \in \mathbb{R}_{+}$, where

$$
\begin{aligned}
Z \triangleq \int_{0}\left|\mu_{s}\right| d s & +\int_{0}^{\cdot}\left(\sigma_{s}^{2} c_{s}+\int_{\{|x| \leq 1\}}\left(1 \wedge\left|\sigma_{s} x\right|^{2}\right) F_{s}(d x)\right) d A_{s} \\
& +\int_{0}\left|\sigma_{s} b_{s}+\int\left(\sigma_{s} x \mathbb{I}_{\left\{|x| \leq 1,\left|\sigma_{s} x\right| \leq 1\right\}}-\sigma_{s} h(x)\right) F_{s}(d x)\right| d A_{s}
\end{aligned}
$$

see [12, Theorem III.6.30]. The solution process $X$ is called a strong solution process, if it is adapted to the completed natural filtration of $L$.

(iii) We say that weak (strong) existence holds for the SDE (1.2), if there exists a driving system which supports a (strong) solution process.

(iv) We say that strong uniqueness holds for the SDE (1.2), if on any driving system there exists up to indistinguishability at most one solution process.

(v) A probability measure $Q$ on $(\mathbb{D}, \mathcal{D})$ (resp. on $(\mathbb{D} \times \mathbb{D}, \mathcal{D} \otimes \mathcal{D})$ ) is called a (joint) solution measure for the $\operatorname{SDE}(1.2)$, if there exists a driving system $(\mathbb{B}, L)$ which supports a solution process $X$ such that $Q$ is the law of $X$ (resp. the law of $(X, L)$ ).

(vi) We say that weak (joint) uniqueness holds for the SDE (1.2), if there exists at most one (joint) solution measure.

Since laws of SIIs are completely characterized by their local characteristics, see [12, Theorem II.4.25], the following theorem follows from [11, Corollary 8.4].

Theorem 2.2. For the SDE (1.2) the following are equivalent:

(i) Strong uniqueness and weak existence holds.

(ii) Weak joint uniqueness and strong existence holds.

In other words, the equivalence of (i) and (iii) in Theorem 1.1 holds. In the next section we will prove the following:

Theorem 2.3. For the SDE (1.2) weak uniqueness implies weak joint uniqueness.

Together with Theorem 2.2, this result implies Theorem 1.1.

Remark 2.4. [11, Theorem 7.2] shows that the existence of a strong solution on some driving system even implies the existence of a strong solution process on every driving system.

We end this section with comments on possible applications. It is interesting to prove analytic conditions implying strong uniqueness. For Brownian SDEs many such conditions are known, see e.g. [8]. There are less results for SDEs with jumps, see e.g. $[3,18]$ for some recent results. Due to Theorem 1.1, instead of verifying strong uniqueness directly, one can deduced it from strong existence and weak uniqueness, where latter is in general better understood than strong uniqueness, see e.g. [16] for some recent results for Lévy driven SDEs.

As a question for future research, it would be interesting whether one can prove analytic conditions for strong existence without using the Yamada-Watanabe theorem, i.e. strong uniqueness. As pointed out above, this could lead to new results for strong uniqueness.

We stress that, in general, boundedness, ellipticity and continuity ${ }^{2}$ conditions do not suffice for strong existence. For the Brownian case a counterexample is given in [1] and for SDEs driven by certain stable Lévy processes a counterexample is given in [4], see also [2] and the comment on p. 10 in [3]. For the Brownian case let us also mention an

\footnotetext{
${ }^{2}$ even local Hölder continuity with too small exponent
} 
example of an SDE with degenerate diffusion coefficient and without strong solution. For $\mu>0$ consider the system

$$
d X_{t}=\mathbb{I}_{\left\{X_{t}>0\right\}} d W_{t}+\frac{1}{2} L_{t}^{0}(X), \quad \mathbb{I}_{\left\{X_{t}=0\right\}} d t=\frac{1}{2 \mu} d L_{t}^{0}(X), \quad X_{0}=x_{0} \in \mathbb{R}_{+},
$$

where $W$ is a Brownian motion and $L^{0}(X)$ denotes the semimartingale (right) local time of $X$ in zero. A solution process $X$ to this system is called Brownian motion with sticky reflecting in the origin. By [7, Theorems 5, 6], the system (2.2) is equivalent to the single equation

$$
d X_{t}=\mu \mathbb{I}_{\left\{X_{t}=0\right\}} d t+\mathbb{I}_{\left\{X_{t}>0\right\}} d W_{t}, \quad X_{0}=x_{0} \in \mathbb{R}_{+},
$$

which satisfies weak existence and joint weak uniqueness, but not strong existence. Consequently, by the Yamada-Watanabe theorem, the SDE (2.3) does not satisfy strong uniqueness. In [7, Section 3.2] it has been noted that strong uniqueness could be disproved in a direct manner by constructing two distinguishable solution processes, which, by the dual Yamada-Watanabe theorem, outlines an alternative proof for [7, Theorem 6]. It has also been noted that Cherny's [5] version of Theorem 2.3 would shorten the proof of [7, Theorem 5].

\section{Proof of Theorem 2.3}

Assume that the SDE (1.2) satisfies weak uniqueness and let $X$ be a solution process on a driving system $\left(\left(\Omega^{*}, \mathcal{F}^{*},\left(\mathcal{F}_{t}^{*}\right)_{t \geq 0}, P^{*}\right), L\right)$. Furthermore, let $\left(\left(\Omega^{o}, \mathcal{F}^{o},\left(\mathcal{F}_{t}^{o}\right)_{t \geq 0}, P^{o}\right), U\right)$ be a second driving system, set

$$
\Omega \triangleq \Omega^{*} \times \Omega^{o}, \quad \mathcal{F} \triangleq \mathcal{F}^{*} \otimes \mathcal{F}^{o}, \quad P \triangleq P^{*} \otimes P^{o},
$$

and define $\mathcal{F}_{t}$ to be the $P$-completion of the $\sigma$-field $\bigcap_{s>t}\left(\mathcal{F}_{s}^{*} \otimes \mathcal{F}_{s}^{o}\right)$. In the following $\mathbb{B}=\left(\Omega, \mathcal{F},\left(\mathcal{F}_{t}\right)_{t \geq 0}, P\right)$ will be our underlying filtered probability space. We extend $X, L$ and $U$ to $\mathbb{B}$ by setting

$$
X\left(\omega^{*}, \omega^{o}\right) \equiv X\left(\omega^{*}\right), \quad L\left(\omega^{*}, \omega^{o}\right) \equiv L\left(\omega^{*}\right), \quad U\left(\omega^{*}, \omega^{o}\right) \equiv U\left(\omega^{o}\right)
$$

for $\left(\omega^{*}, \omega^{o}\right) \in \Omega$. Due to the results in [10, Section $\left.\left.10.2 \mathrm{~b}\right)\right],(\mathbb{B}, L)$ and $(\mathbb{B}, U)$ are driving systems and $X$ is a solution process on $(\mathbb{B}, L)$. Next, we define a semimartingale $V$ by

$$
d V_{t} \triangleq \mathbb{I}_{\left\{\sigma_{t}(X) \neq 0\right\}} d U_{t}+\mathbb{I}_{\left\{\sigma_{t}(X)=0\right\}} d L_{t} .
$$

The following lemma is proven after the proof of Theorem 2.3 is complete.

Lemma 3.1. The process $V$ is an SII with the same local characteristics as L. Moreover, $V$ is independent of $X$.

Since

$$
d L_{t}=\frac{\mathbb{I}_{\left\{\sigma_{t}(X) \neq 0\right\}}\left(d X_{t}-\mu_{t}(X) d t\right)}{\sigma_{t}(X)}+\mathbb{I}_{\left\{\sigma_{t}(X)=0\right\}} d V_{t},
$$

the distribution of $(X, L)$ is completely determined by the distribution of $(X, V)$ and Lemma 3.1 yields that weak joint uniqueness is implied by weak uniqueness. The proof of Theorem 2.3 is complete.

Remark 3.2. In case $\sigma$ is non-degenerate, i.e. $\sigma \neq 0$, it is clear that $V=U$ and Lemma 3.1 becomes trivial. In particular, for the key argument it is not necessary to introduce the auxilliary process $U$, see (1.1) in the introduction.

It remains to prove Lemma 3.1: 
Proof of Lemma 3.1: Step 1. Recall that the local characteristics of $L$ are denoted by $(b, c, F ; A)$. Our first step is to show that $V$ is an SII with local characteristics $(b, c, F ; A)$ by computing its semimartingale characteristics $\left(B^{V}, C^{V}, \nu^{V}\right)$. We start with the first characteristic $B^{V}$. For $h^{*}(x) \triangleq x-h(x)$, we have

$$
\begin{aligned}
\sum_{s \leq \cdot} h^{*}\left(\Delta V_{s}\right) & =\sum_{s \leq \cdot} h^{*}\left(\mathbb{I}_{\left\{\sigma_{s}(X) \neq 0\right\}} \Delta U_{s}+\mathbb{I}_{\left\{\sigma_{s}(X)=0\right\}} \Delta L_{s}\right)\left(\mathbb{I}_{\left\{\sigma_{s}(X) \neq 0\right\}}+\mathbb{I}_{\left\{\sigma_{t}(X)=0\right\}}\right) \\
& =\sum_{s \leq \cdot} h^{*}\left(\Delta U_{s}\right) \mathbb{I}_{\left\{\sigma_{s}(X) \neq 0\right\}}+\sum_{s \leq .} h^{*}\left(\Delta L_{s}\right) \mathbb{I}_{\left\{\sigma_{s}(X)=0\right\}} .
\end{aligned}
$$

The definition of the first semimartingale characteristic shows that the processes

$$
U-\sum_{s \leq \cdot} h^{*}\left(\Delta U_{s}\right)-\int_{0}^{\cdot} b_{s} d A_{s}, \quad L-\sum_{s \leq \cdot} h^{*}\left(\Delta L_{s}\right)-\int_{0}^{\cdot} b_{s} d A_{s}
$$

are local martingales. Hence, the process

$$
\begin{array}{r}
V-\sum_{s \leq .} h^{*}\left(\Delta V_{s}\right)-\int_{0} b_{s} d A_{s}=\mathbb{I}_{\{\sigma(X) \neq 0\}} \cdot\left(U-\sum_{s \leq \cdot} h^{*}\left(\Delta U_{s}\right)-\int_{0} b_{s} d A_{s}\right) \\
+\mathbb{I}_{\{\sigma(X)=0\}} \cdot\left(L-\sum_{s \leq \cdot} h^{*}\left(\Delta L_{s}\right)-\int_{0} b_{s} d A_{s}\right)
\end{array}
$$

is a local martingale, too. This implies that $d B_{t}^{V}=b_{t} d A_{t}$. Next, we compute the second characteristic $C^{V}$. Using that $d V_{t}^{c}=\mathbb{I}_{\left\{\sigma_{t}(X) \neq 0\right\}} d U_{t}^{c}+\mathbb{I}_{\left\{\sigma_{t}(X)=0\right\}} d L_{t}^{c}$, we obtain

$$
d C_{t}^{V}=d\left[V^{c}, V^{c}\right]_{t}=\mathbb{I}_{\left\{\sigma_{t}(X) \neq 0\right\}} d\left[U^{c}, U^{c}\right]_{t}+\mathbb{I}_{\left\{\sigma_{t}(X)=0\right\}} d\left[L^{c}, L^{c}\right]_{t}=c_{t} d A_{t} .
$$

Finally, we compute the third characteristic $\nu^{V}$. For every bounded stopping time $T$ and any bounded Borel function $G: \mathbb{R} \rightarrow \mathbb{R}$ which vanishes in a neighborhood of the origin, we obtain

$$
\begin{aligned}
E\left[\sum_{s \leq T} G\left(\Delta V_{s}\right)\right] & =E\left[\sum_{s \leq T}\left(G\left(\Delta U_{s}\right) \mathbb{I}_{\left\{\sigma_{s}(X) \neq 0\right\}}+G\left(\Delta L_{s}\right) \mathbb{I}_{\left\{\sigma_{s}(X)=0\right\}}\right)\right] \\
& =E\left[\int_{0}^{T} \int\left(G(x) \mathbb{I}_{\left\{\sigma_{s}(X) \neq 0\right\}}+G(x) \mathbb{I}_{\left\{\sigma_{s}(X)=0\right\}}\right) F_{s}(d x) d A_{s}\right] \\
& =E\left[\int_{0}^{T} \int G(x) F_{s}(d x) d A_{s}\right] .
\end{aligned}
$$

This shows that $\nu^{V}(d x, d t)=F_{t}(d x) d A_{t}$. In summary, the process $V$ is an SII with local characteristics $(b, c, F ; A)$.

Step 2. In this step we show that $V$ and $X$ are independent. Hereby, we borrow ideas used in the proof of [9, Theorem 4.10.1]. We start with three preparatory lemmata.

Lemma 3.3. The bivariate process $(U, L)$ is a two-dimensional SII with local characteristics $\left(b^{(U, L)}, c^{(U, L)}, F^{(U, L)} ; A\right)$ given by

$$
b^{(U, L)}=(b, b), \quad c^{(U, L)}=\left(\begin{array}{cc}
c & 0 \\
0 & c
\end{array}\right), \quad F^{(U, L)}(d x, d y)=F(d x) \delta_{0}(d y)+\delta_{0}(d x) F(d y) .
$$

Proof. It is clear that $(U, L)$ is a two-dimensional semimartingale. Fix $0 \leq s<t$. By the construction of $\mathbb{B}$ and a monotone class argument, we see that $\left(U_{t}-U_{s}, L_{t}-L_{s}\right)$ is independent of $\mathcal{F}_{s}^{*} \otimes \mathcal{F}_{s}^{o}$. Let $f: \mathbb{R}^{2} \rightarrow \mathbb{R}_{+}$be bounded and continuous and take $G \in \mathcal{F}_{s}$. For every $\varepsilon \in(0, t-s)$ we have

$$
E\left[f\left(U_{t}-U_{s+\varepsilon}, L_{t}-L_{s+\varepsilon}\right) \mathbb{I}_{G}\right]=E\left[f\left(U_{t}-U_{s+\varepsilon}, L_{t}-L_{s+\varepsilon}\right)\right] P(G) .
$$


Thus, using that $U$ and $L$ have right-continuous paths, that for every closed set $F \subseteq \mathbb{R}^{2}$ there exists a uniformly bounded sequence $\left(f_{n}\right)_{n \in \mathbb{N}}$ of non-negative continuous functions such that $f_{n}(x) \rightarrow \mathbb{I}_{F}(x)$, and a monotone class argument, we conclude that $(U, L)$ has independent increments relative to the filtration $\left(\mathcal{F}_{t}\right)_{t \geq 0}$. Finally, the structure of the characteristics follows from the independence of $U$ and $L$, the Lévy-Khinchine formula, see [12, Theorem II.4.15], and the uniqueness lemma [12, Lemma II.2.44].

For $f \in C_{b}^{2}(\mathbb{R})$, which is the space of bounded twice continuously differentiable functions with bounded first and second derivative, and $(x, t) \in \mathbb{R} \times \mathbb{R}_{+}$we set

$$
\mathcal{L} f(x, t) \triangleq b_{t} f^{\prime}(x)+\frac{c_{t}}{2} f^{\prime \prime}(x)+\int\left(f(x+y)-f(x)-h(y) f^{\prime}(x)\right) F_{t}(d y) .
$$

The next lemma is a martingale characterization for SIIs.

Lemma 3.4. On a filtered probability space with right-continuous filtration, let $Y$ be an adapted process with càdlàg paths and starting value $Y_{0}=0$. Then, the following are equivalent:

(i) $Y$ is an SII with local characteristics $(b, c, F ; A)$.

(ii) For all $f \in C_{b}^{2}(\mathbb{R})$ with $\inf _{x \in \mathbb{R}} f(x)>0$ the process

$$
M^{f} \triangleq \frac{f(Y)}{f(0)} \exp \left(-\int_{0}^{\cdot} \frac{\mathcal{L} f\left(Y_{s}, s\right) d A_{s}}{f\left(Y_{s}\right)}\right)
$$

is a martingale.

Proof. Suppose that (i) holds and let $f \in C_{b}^{2}(\mathbb{R})$ with $\inf _{x \in \mathbb{R}} f(x)>0$. Integration by parts yields that

$$
d M_{t}^{f}=\frac{1}{f(0)} \exp \left(-\int_{0}^{t} \frac{\mathcal{L} f\left(Y_{s}, s\right) d A_{s}}{f\left(Y_{s}\right)}\right)\left(d f\left(Y_{t}\right)-\mathcal{L} f\left(Y_{t}, t\right) d A_{t}\right) .
$$

By [12, Theorem II.2.42], the process $f(Y)-\int_{0}^{*} \mathcal{L} f\left(Y_{s}, s\right) d A_{s}$ is a local martingale. Thus, also $M^{f}$ is a local martingale. As $h$ is a truncation function, there exists a constant $\varepsilon>0$ such that $h(x)=x$ whenever $x \in(-\varepsilon, \varepsilon)$. Furthermore, by Taylor's theorem,

$$
\left|f(x+y)-f(x)-f^{\prime}(x) y\right| \leq \frac{1}{2}\left\|f^{\prime \prime}\right\|_{\infty}|y|^{2}, \quad y \in \mathbb{R} .
$$

Thus, $\int_{0}^{\cdot} \int\left(1 \wedge|y|^{2}\right) F_{s}(d y) d A_{s}<\infty$ implies that the process $M^{f}$ is bounded on bounded time intervals and consequently, a martingale. We conclude that (ii) holds.

Conversely, assume that (ii) holds. Again, let $f \in C_{b}^{2}(\mathbb{R})$ with $\inf _{x \in \mathbb{R}} f(x)>0$. Then, integration by parts yields that

$$
d f\left(Y_{t}\right)-\mathcal{L} f\left(Y_{t}, t\right) d A_{t}=f(0) \exp \left(\int_{0}^{t} \frac{\mathcal{L} f\left(Y_{s}, s\right) d A_{s}}{f\left(Y_{s}\right)}\right) d M_{t}^{f} .
$$

Hence, $f(Y)-\int_{0} \mathcal{L} f\left(Y_{s}, s\right) d A_{s}$ is a local martingale. Applying this observation with $f(x)=2+\sin (u x)$ and $f(x)=2+\cos (u x)$ for $u \in \mathbb{R}$ yields that b) of [12, Theorem II.2.42] holds and (i) follows.

Next, we also give a martingale characterization for the set of solution measures to the $\operatorname{SDE}(1.2)$. For $g \in C_{b}^{2}(\mathbb{R})$ and $(\omega, s) \in \mathbb{D} \times \mathbb{R}_{+}$we set

$$
\begin{aligned}
\mathcal{K} g(\omega, s) \triangleq & \sigma_{s}(\omega) b_{s} g^{\prime}(\omega(s-))+\frac{\sigma_{s}^{2}(\omega) c_{s}}{2} g^{\prime \prime}(\omega(s-)) \\
& +\int\left(g\left(\omega(s-)+\sigma_{s}(\omega) x\right)-g(\omega(s-))-\sigma_{s}(\omega) h(x) g^{\prime}(\omega(s-))\right) F_{s}(d x) .
\end{aligned}
$$


Similar to the proof of [10, Theorem 14.80], the following lemma follows from representation results for continuous local martingales and integer valued random measures. We refer to [17] for an approach not relying on representation theorems.

Lemma 3.5. A probability measure $Q$ on $(\mathbb{D}, \mathcal{D})$ is a solution measure to the $S D E(1.2)$ if and only if $Q\left(\mathrm{X}_{0}=x_{0}\right)=1, Z$ as defined in (2.1) is $Q$-a.s. finite, and for all $g \in C_{b}^{2}(\mathbb{R})$ the process

$$
K^{g} \triangleq g(\mathbf{X})-g\left(x_{0}\right)-\int_{0}^{\cdot} \mu_{s}(\mathbf{X}) g^{\prime}\left(\mathbf{X}_{s}\right) d s-\int_{0}^{\cdot} \mathcal{K} g(\mathbf{X}, s) d A_{s}
$$

is a local $\left(\mathbf{D}^{Q}, Q\right)$-martingale. Furthermore, for every solution process $X$ to the SDE (1.2) the process $K^{g} \circ X$ is a local martingale on the corresponding driving system.

Proof. Let $X$ be a solution process to the SDE (1.2) and denote its law by $Q$. Then, [12, Theorem II.2.42] and [15, Lemma 3] yield that the process $K^{g} \circ X$ is a local martingale for each $g \in C_{b}^{2}(\mathbb{R})$. Due to [10, Remark 10.40] this local martingale property transfers to the canonical space, i.e. $K^{g}$ is a local $\left(\mathbf{D}^{Q}, Q\right)$-martingale. Hence, the final claim and the only if implication are proven.

Conversely, assume that $Q$ is as in the if implication. Recalling again [15, Lemma 3], we deduce from [12, Theorems II.2.34, II.2.42] and [14, Theorems 1, 2] that, possibly on a standard extension of the canonical space endowed with $Q$, there exists a continuous local martingale $L^{c}$ with quadratic variation $\left[L^{c}, L^{c}\right]=\int_{0}^{\cdot} c_{s} d A_{s}$ and an integer valued random measure $\mu(d x, d t)$ with intensity measure $\nu(d x, d t)=F_{t}(d x) d A_{t}$ such that

$$
\begin{aligned}
d \mathbf{X}_{t}=\mu_{t}(\mathbf{X}) d t & +\sigma_{t}(\mathbf{X})\left(b_{t}+\int\left(x \mathbb{I}_{\left\{\left|\sigma_{t}(\mathbf{X}) x\right| \leq 1\right\}}-h(x)\right) F_{t}(d x)\right) d A_{t} \\
& +\sigma_{t}(\mathbf{X}) d L_{t}^{c}+\int \sigma_{t}(\mathbf{X}) x \mathbb{I}_{\left\{\left|\sigma_{t}(\mathbf{X}) x\right| \leq 1\right\}}(\mu-\nu)(d x, d t) \\
& +\int \sigma_{t}(X) x \mathbb{I}_{\left\{\left|\sigma_{t}(\mathbf{X}) x\right|>1\right\}} \mu(d x, d t)
\end{aligned}
$$

Rearranging yields that $d \mathbf{X}_{t}=\mu_{t}(\mathbf{X}) d t+\sigma_{t}(\mathbf{X}) d L_{t}$, where

$$
d L_{t} \triangleq b_{t} d A_{t}+d L_{t}^{c}+\int h(x)(\mu-\nu)(d x, d t)+\int(x-h(x)) \mu(d x, d t) .
$$

It is easy to see that $L$ is an SII with local characteristics $(b, c, F ; A)$ and consequently, the if implication holds. The proof is complete.

We are in the position to prove the independence of $V$ and $X$. Take $f, g \in C_{b}^{2}(\mathbb{R})$ with $\inf _{x \in \mathbb{R}} f(x)>0$ and define $M^{f}$ and $K^{g}$ as in (3.1) and (3.3) with $Y$ replaced by $V$ and $\mathrm{X}$ replaced by $X$. As shown in Step $1, V$ is an SII with local characteristics $(b, c, F ; A)$. Hence, $M^{f}$ is a martingale by Lemma 3.4. Similarly, because $X$ is a solution process to the SDE (1.2), $K^{g}$ is a local martingale by Lemma 3.5. We now show that $\left[M^{f}, K^{g}\right]=0$. Recalling (3.2) and the proof of a) $\Rightarrow$ c) in [12, Theorem II.2.42], we see that

$$
\left(M^{f}\right)^{c}=\frac{f^{\prime}(V)}{f(0)} \exp \left(-\int_{0}^{\cdot} \frac{\mathcal{L} f\left(V_{s}, s\right) d A_{s}}{f\left(V_{s}\right)}\right) \cdot V^{c} .
$$

Similarly, $\left(K^{g}\right)^{c}=g^{\prime}(X) \sigma(X) \cdot L^{c}$. Since $\left[U^{c}, L^{c}\right]=0$ by Lemma 3.3, we obtain

$$
d\left[V^{c}, \sigma(X) \cdot L^{c}\right]_{t}=\sigma_{t}(X) \mathbb{I}_{\left\{\sigma_{t}(X) \neq 0\right\}} d\left[U^{c}, L^{c}\right]_{t}+\sigma_{t}(X) \mathbb{I}_{\left\{\sigma_{t}(X)=0\right\}} d\left[L^{c}, L^{c}\right]_{t}=0,
$$

which implies that $\left[\left(M^{f}\right)^{c},\left(K^{g}\right)^{c}\right]=0$. Using the formula for the third characteristic of $(U, L)$ as given in Lemma 3.3 yields that

$$
E\left[\sum_{s \leq .}\left|\Delta U_{s} \Delta L_{s}\right|\right]=\int_{0}^{\cdot} \int|x y| F_{s}^{(U, L)}(d x, d y) d A_{s}=0 .
$$


In other words, $U$ and $L$ cannot jump at the same time, which implies that

$$
\sigma(X) \neq 0, \Delta L \neq 0 \Longrightarrow \Delta V=\mathbb{I}_{\{\sigma(X) \neq 0\}} \Delta U+\mathbb{I}_{\{\sigma(X)=0\}} \Delta L=0 .
$$

Hence, we obtain

$$
\sigma(X) \neq 0, \Delta L \neq 0 \Longrightarrow \Delta M^{f}=\frac{\Delta f(V)}{f(0)} \exp \left(-\int_{0} \frac{\mathcal{L} f\left(V_{s}, s\right) d A_{s}}{f\left(V_{s}\right)}\right)=0 .
$$

Furthermore, because $\Delta X=\sigma(X) \Delta L$, we have

$$
\sigma(X)=0 \text { or } \Delta L=0 \Longrightarrow \Delta K^{g}=\Delta g(X)=0 .
$$

Putting these pieces together, we conclude that $\sum_{s \leq} \Delta M_{s}^{f} \Delta K_{s}^{g}=0$ and therefore

$$
\left[M^{f}, K^{g}\right]=\left[\left(M^{f}\right)^{c},\left(K^{g}\right)^{c}\right]+\sum_{s \leq .} \Delta M_{s}^{f} \Delta K_{s}^{g}=0 .
$$

For $n \in \mathbb{N}$ we define

$$
T_{n} \triangleq \inf \left(t \in \mathbb{R}_{+}:\left|K_{t}^{g}\right|>n\right), \quad K^{g, n} \triangleq K_{\cdot \wedge T_{n}}^{g} .
$$

As $K^{g}$ has bounded jumps, $K^{g, n}$ is bounded on bounded time intervals. Thus, by integration by parts, $\left[M^{f}, K^{g, n}\right]=\left[M^{f}, K^{g}\right]_{. \wedge T_{n}}=0$ yields that the process $M^{f} K^{g, n}$ is a martingale which is bounded on bounded time intervals.

Now, fix a bounded stopping time $S$ and define a measure $Q$ as follows:

$$
Q(G) \triangleq E^{P}\left[M_{S}^{f} \mathbb{I}_{G}\right], \quad G \in \mathcal{F} .
$$

Clearly, as $M^{f}$ is a $P$-martingale with $M_{0}^{f}=1, Q$ is a probability measure by the optional stopping theorem. Moreover, because $Q \sim P$, we have $Q\left(X_{0}=x_{0}\right)=1$ and $Q$-a.s. $Z_{t} \circ X<\infty$ for all $t \in \mathbb{R}_{+}$, where $Z$ is defined in (2.1). As $M^{f}, K^{g, n}$ and $M^{f} K^{g, n}$ are $P$-martingales, we obtain for every bounded stopping time $T$ that

$$
\begin{aligned}
E^{Q}\left[K_{T}^{g, n}\right] & =E^{P}\left[M_{S}^{f} K_{T}^{g, n}\right] \\
& =E^{P}\left[M_{S}^{f} \mathbb{I}_{\{S \leq T\}} E^{P}\left[K_{T}^{g, n} \mid \mathcal{F}_{S \wedge T}\right]+K_{T}^{g, n} \mathbb{I}_{\{T<S\}} E^{P}\left[M_{S}^{f} \mid \mathcal{F}_{S \wedge T}\right]\right] \\
& =E^{P}\left[M_{S}^{f} K_{S \wedge T}^{g, n} \mathbb{I}_{\{S \leq T\}}+K_{T}^{g, n} M_{S \wedge T}^{f} \mathbb{I}_{\{T<S\}}\right] \\
& =E^{P}\left[M_{S \wedge T}^{f} K_{S \wedge T}^{g, n}\right]=0 .
\end{aligned}
$$

Thus, because the stopping time $T$ was arbitrary, $K^{g, n}$ is a $Q$-martingale. Furthermore, since $g$ was arbitrary and $T_{n} \nearrow \infty$ as $n \rightarrow \infty$, Lemma 3.5 and [10, Remark 10.40] show that the push-forward $Q \circ X^{-1}$ is a solution measure to the SDE (1.2). Consequently, by the weak uniqueness assumption, $P \circ X^{-1}=Q \circ X^{-1}$.

Next, take $F \in \sigma\left(X_{t}, t \in \mathbb{R}_{+}\right)$such that $P(F)>0$ and set

$$
Q^{*}(G) \triangleq \frac{P(G \cap F)}{P(F)}, \quad G \in \mathcal{F} .
$$

Using that $P(F)=Q(F)$, we obtain that

$$
E^{Q^{*}}\left[M_{S}^{f}\right]=\frac{Q(F)}{P(F)}=1 .
$$

Thus, as the stopping time $S$ was arbitrary, $M^{f}$ is a $Q^{*}$-martingale. Since $f$ was arbitrary, we deduce from Lemma 3.4 that $Q^{*} \circ V^{-1}=P \circ V^{-1}$ and consequently, for every $G \in \sigma\left(V_{t}, t \in \mathbb{R}_{+}\right)$

$$
P(G \cap F)=Q^{*}(G) P(F)=P(G) P(F) .
$$

As this equality holds trivially for all $F \in \sigma\left(X_{t}, t \in \mathbb{R}_{+}\right)$with $P(F)=0$, we conclude that $V$ and $X$ are independent. The proof is complete. 


\section{References}

[1] M. T. Barlow. One dimensional stochastic differential equations with no strong solution. Journal of the London Mathematical Society, s2-26(2):335-347, 1982. MR-0675177

[2] R. F. Bass. Stochastic differential equations driven by symmetric stable processes. Séminaire de probabilités de Strasbourg, 36:302-313, 2002. MR-1971592

[3] R. F. Bass. Stochastic differential equations with jumps. Probability Surveys, 1:1-19, 2004. MR-2095564

[4] R. F. Bass, K. Burdzy, and Z.-Q. Chen. Stochastic differential equations driven by stable processes for which pathwise uniqueness fails. Stochastic Processes and their Applications, 111(1):1-15, 2004. MR-2049566

[5] A. S. Cherny. On the uniqueness in law and the pathwise uniqueness for stochastic differential equations. Theory of Probability \& Its Applications, 46(3):406-419, 2002.

[6] H.-J. Engelbert. On the theorem of T. Yamada and S. Watanabe. Stochastics and Stochastic Reports, 36(3-4):205-216, 1991. MR-1128494

[7] H.-J. Engelbert and G. Peskir. Stochastic differential equations for sticky Brownian motion. Stochastics, 86(6):993-1021, 2014. MR-3271518

[8] H.-J. Engelbert and W. Schmidt. Strong Markov continuous local martingales and solutions of one-dimensional stochastic differential equations (part III). Mathematische Nachrichten, 151(1):149-197, 1991. MR-1121203

[9] S. N. Ethier and T. G. Kurtz. Markov Processes: Characterization and Convergence. Wiley, 2005. MR-0838085

[10] J. Jacod. Calcul stochastique et problèmes de martingales. Springer Berlin Heidelberg New York, 1979. MR-0542115

[11] J. Jacod. Weak and strong solutions of stochastic differential equations. Stochastics, 3(14):171-191, 1980. MR-0573202

[12] J. Jacod and A. N. Shiryaev. Limit Theorems for Stochastic Processes. Springer Berlin Heidelberg, 2nd edition, 2003. MR-1943877

[13] J. Jacod. Two dependent Poisson processes whose sum is still a Poisson process. Journal of Applied Probability, 12(1):170-172, 1975. MR-0370750

[14] Y. M. Kabanov, R. S. Liptser, and A. N. Shiryaev. On the representation of integral-valued random measures and local martingales by means of random measures with deterministic compensators. Mathematics of the USSR-Sbornik, 39(2):267-280, 1981. MR-0564354

[15] J. Kallsen and A. N. Shiryaev. Time change representation of stochastic integrals. Theory of Probability and its Applications, 46(3):522-528, 2002. MR-1978671

[16] F. Kühn. On martingale problems and Feller processes. Electronic Journal of Probability, 23(13):1-18, 2018. MR-3771750

[17] T. G. Kurtz. Equivalence of stochastic equations and martingale problems. Stochastic Analysis 2010, Ed. D. Crisan, Springer Berlin Heidelberg, 113-130, 2011. MR-2789081

[18] Z. Li and F. Pu. Strong solutions of jump-type stochastic equations. Electronic Communications in Probability, 17(33):1-13, 2012. MR-2965746

[19] H. Qiao. A theorem dual to Yamada-Watanabe theorem for stochastic evolution equations. Stochastics and Dynamics, 10(03):367-374, 2010. MR-2671381

[20] M. Rehmeier. On Cherny's results in infinite dimensions: a theorem dual to Yamada-Watanabe. Stochastics and Partial Differential Equations: Analysis and Computations, 9:33-70, 2021. MR-4218787

[21] S. Tappe. The dual Yamada-Watanabe theorem for mild solutions to stochastic partial differential equations. arXiv:2006.13038v1, 2020.

[22] T. Yamada and S. Watanabe. On the uniqueness of solutions of stochastic differential equations. Journal of Mathematics of Kyoto University, 11(1):155-167, 1971. MR-0278420

[23] H. Zhao, C. Hu, and S. Xu. Equivalence of uniqueness in law and joint uniqueness in law for SDEs driven by Poisson processes. Applied Mathematics, 7(8):784-792, 2016.

Acknowledgments. The author thanks the anonymous referee for many helpful comments. 